Provided for non-commercial research and education use. Not for reproduction, distribution or commercial use.

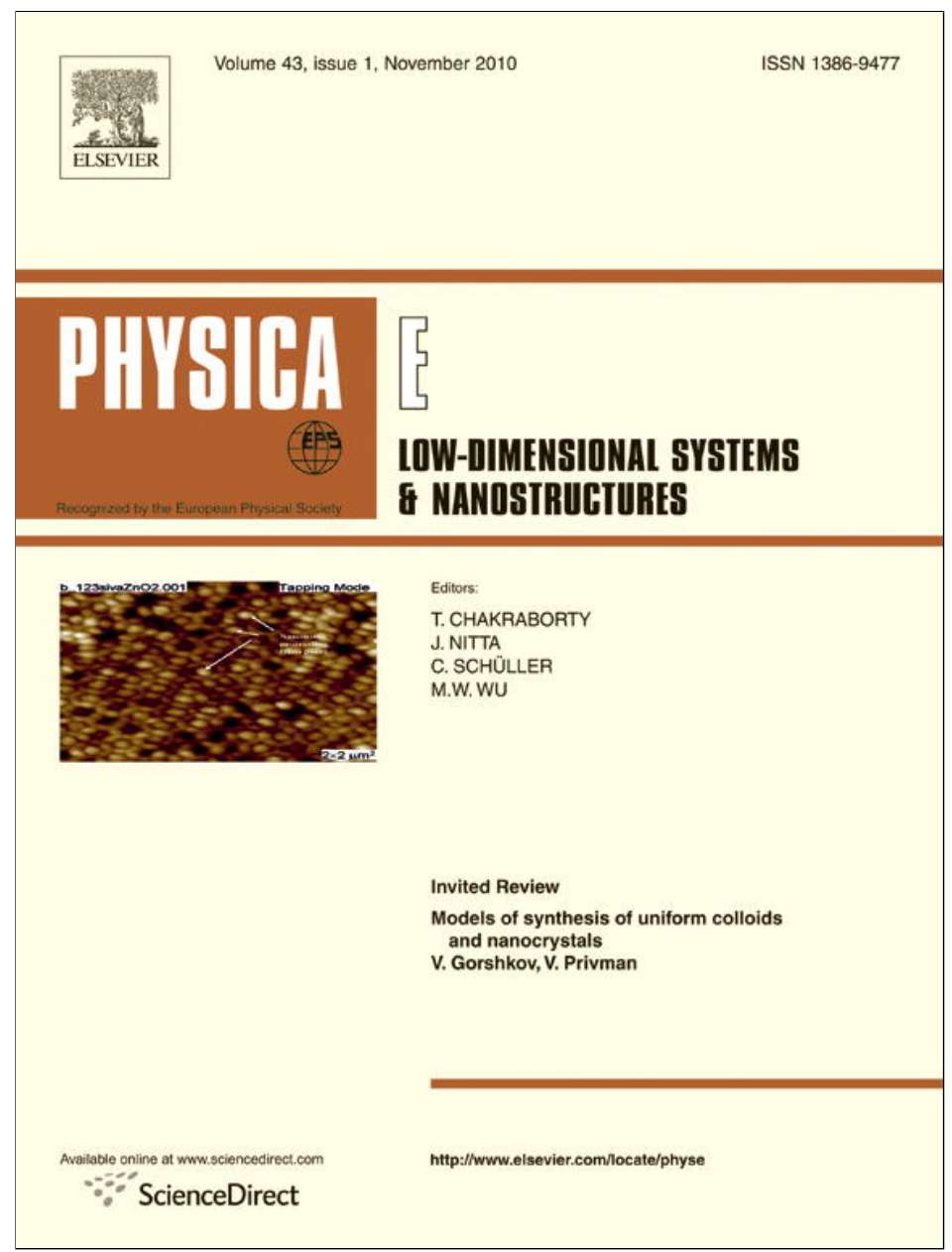

This article appeared in a journal published by Elsevier. The attached copy is furnished to the author for internal non-commercial research and education use, including for instruction at the authors institution and sharing with colleagues.

Other uses, including reproduction and distribution, or selling or licensing copies, or posting to personal, institutional or third party websites are prohibited.

In most cases authors are permitted to post their version of the article (e.g. in Word or Tex form) to their personal website or institutional repository. Authors requiring further information regarding Elsevier's archiving and manuscript policies are encouraged to visit:

http://www.elsevier.com/copyright 


\title{
Nonlocal effects in the longitudinal vibration of double-nanorod systems
}

\author{
T. Murmu, S. Adhikari* \\ School of Engineering, Swansea University, Singleton Park, Swansea SA2 8PP, Wales, UK
}

\section{A R T I C L E I N F O}

\section{Article history:}

Received 1 July 2010

Received in revised form

20 August 2010

Accepted 23 August 2010

Available online 27 August 2010

\begin{abstract}
A B S T R A C T
This paper presents an investigation on the longitudinal vibration of a double-nanorod-system (DNRS). The double-nanorod-systems are important in nanooptomechanical systems (NOMS). For the development of the governing equations, Eringen's nonlocal elasticity is utilized. It is assumed that the two nanorods of the DNRS are coupled by longitudinally directed distributed springs. An analytical method is developed for solving the nonlocal frequencies of longitudinally vibrating DNRS. Clampedclamped and clamped-free boundary conditions are employed and their explicit relationships are derived. Numerical studies are carried out for coupled double-carbon-nanotube-rod system. This study highlights that the nonlocal effect considerably influences the axial vibration of DNRS. The results obtained in this paper can be useful for the study of axially vibrating complex multiple-nanobeam system in NOMS.
\end{abstract}

(c) 2010 Elsevier B.V. All rights reserved.

\section{Introduction}

Nanomaterials are observed to have promising mechanical, chemical, electrical, optical and electronic properties [1-3]. Such nanomaterials include carbon nanotubes, graphene sheet, zinc oxide $(\mathrm{ZnO})$ nanowires, boron nitride $(\mathrm{BN})$ nanotubes and nanosheets. The excellent properties of these nanomaterials have led to its multiple usages in the field of nanoelectronics, nanodevices, nanosensors, nanooscillators, nanoactuators, nanobearings, and micromechanical resonators, transporter of drugs, hydrogen storage, electrical batteries, nanocomposites and nanooptomechanical systems (NOMS). However, for further effective potential applications of theses nanostructures, proper physical, chemical and mechanical understanding of the nanomaterials is essential.

Nanomaterials are the base material of many nanoscale objects. Nanoscale objects are referred to as nanostructures. Recently various one-dimensional nanostructures have been realized. They include nanodots, nanorods, nanowires, nanobelts, nanotubes, nanobridges and nanonails, nanowalls, nanohelices, seamless nanorings. Among all the one-dimensional nanostructures, nanotubes, nanorods and nanowires are widely studied. This is because of the easy material formation and device applications.

Nanorods [4] are one-dimensional nanoobjects ranging from 1 to $3000 \mathrm{~nm}$ (Fig. 1). These miniscule dimension nanorods or nanowires can be grown from various methods. The popular

\footnotetext{
* Corresponding author. Tel.: +441792 602088; fax: +44 1792295676.

E-mail address: S.Adhikari@swansea.ac.uk (S. Adhikari).
}

methods include (i) vapour phase synthesis [5], (ii) metal-organic chemical vapour deposition [6], (iii) hydrothermal synthesis [7]. Nanorods have found applications in a variety of nanodevices. These include ultraviolet photodetectors, nanosensors, transistors, diodes, LED arrays.

As controlled experiments in the nanoscale can be difficult, theoretical analysis of nanostructures emerges as an important tool for further understanding. Atomistic methods such as molecular dynamics (MD) and Monte Carlo simulation are computationally prohibitive for nanostructures with large numbers of atoms. Thus simplified analyses have been carried out using the classical continuum mechanics. Though classical continuum models have been able to predict the general behaviour of nanostructures, it lacks the accountability of the effects arising from the small-scale. Consequently the application of these classical continuum models may be questionable in the analysis of nanostructures. Thereby size-dependent continuum based methods are being used increasingly in the modelling of small-sized structures as it offers much faster solutions than molecular dynamics simulations for various nanoengineering problems. Currently research efforts are undergoing to bring in the size-effects within the formulation by modifying the traditional classical mechanics. One widely used size-dependent theory is the nonlocal elasticity theory pioneered by Eringen [8].

The theory of nonlocal elasticity is being increasingly used for efficient analysis of nanostructures viz. nanorods, nanobeams, nanorings, carbon nanotubes, nanoplates and graphenes, etc. Peddieson et al. [9] introduced the nonlocal elasticity theory to nanotechnology. Ref. [9] developed a nonlocal Euler-Bernoulli beam model and studied a case for cantilever beam. Lu [10] utilized Euler-Bernoulli nonlocal beam model and studied the dynamic 


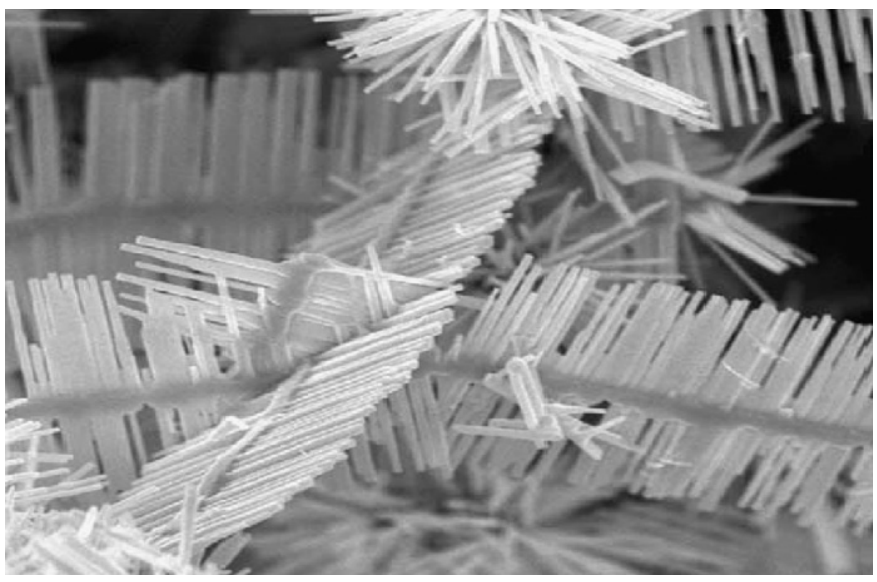

Fig. 1. ZnO nanorods growing on central nanowire cores. The length of these side nanorods ranges from 100 to $3000 \mathrm{~nm}$, while the width of nanowires core is $10-20 \mathrm{~nm}[4]$.

analysis of axially prestressed micro/nanobeam structures based on nonlocal beam theory. Wang et al. [11] studied the elastic buckling phenomenon of micro- and nanorods/tubes based on nonlocal Timoshenko beam theory. A detailed formulation for bending, vibration and buckling of carbon nanotubes was proposed using nonlocal Euler-Bernoulli and Timoshenko theory by Reddy and Pang [12]. Wang and Duan [13] investigated free vibration problem of nanorings/arches using nonlocal elasticity theory. Axial frequencies using nonlocal continuum rod theories were presented by Aydogdu [14], who further proposed a generalised nonlocal beam theory to study bending, buckling and free vibration of nanobeams [15]. Heireche et al. [16] analysed the sound wave propagation in single-walled carbon nanotubes using nonlocal elasticity. Vibration and instability of tubular micro- and nanobeams conveying fluid was studied using the theory of nonlocal elasticity [17]. Yan et al. [18] carried out nonlocal elastic analysis of axially compressed buckling of triple-walled carbon nanotubes under the effect of temperature field. Torsional buckling of carbon nanotubes using nonlocal elasticity shell models were presented by Khademolhosseini et al. [19]. The nonlocal elasticity was also used in the nonlinear vibration analysis of carbon nanotubes [20]. Vibration and buckling of nanoplates is also studied using nonlocal plate theories [21-24].

Nonlocal elasticity accounts for the small-scale effects at the atomistic level. At nanometer scales, size effects often become prominent. Both experimental [25-27] and atomistic simulation results $[28,29]$ have shown a significant 'size-effect' in mechanical properties when the dimensions of these structures become small. In this nonlocal elasticity theory the small-scale effects are captured by assuming that the stress at a point as a function of the strains at all points in the domain [8]. Nonlocal theory considers long-range inter-atomic interaction and yields results dependent on the size of a body. Some of the drawbacks of the classical continuum theory could be efficiently avoided and size-dependent phenomena can be explained by the nonlocal elasticity theory. Further from Chen et al. [30] the nonlocal continuum based models are physically reasonable from the atomistic viewpoint of lattice dynamics and molecular dynamics (MD) simulations.

The majority of the literature on nonlocal elasticity exists pertaining to the free transverse vibration of micro/nanobeams (CNTs), nanoplates (graphene sheets) and nanoshells (CNTS). This is because the transverse modes are more prone to get excited by common types of external excitations. On the contrary, only limited works on nonlocal elasticity have been devoted to the longitudinal vibration of nanobeam and nanoplates. Aydogdu [14] developed a nonlocal elastic rod model, and applied it to investigate the small-scale effect on the axial vibration of clamped-clamped and clamped-free nanorods. Recently, Filiz and Aydogdu [31] applied the axial vibration of nonlocal rod theory to carbon nanotube heterojunction systems. Using nonlocal elasticity theory Murmu and Pradhan [32] studied the free in-plane vibration of nanoplates.

Like the transverse vibration, axial vibration experiments can also be used for the determination of Young's modulus of CNTs. Generally, the flexural modes occur at low frequencies. However vibrating nanobeams (CNTs) may also have longitudinal modes at relatively high frequencies and can be of very practical significance in high operating frequencies. Nanorods when used as electromechanical resonator can be externally excited and exhibit axial vibrations. Further for a moving nanoparticle inside a singlewalled carbon nanotube (SWCNT), the SWCNT generally vibrates both in the transverse and longitudinal directions. The longitudinal vibration is generated because of the existing friction between the outer surface of the moving nanoparticle and the inner surface of the SWCNT. It is also reported [33] that transport measurements on suspended single-wall carbon nanotubes show signatures of phonon assisted tunnelling, influenced by longitudinal vibration (stretching) modes. More recently Chowdhury et al. $[34,35]$ have reported sliding axial modes for multiwalled carbon nanotubes.

Therefore, there is a strong scientific need to gain an understanding of the longitudinal vibration of complex nanorods system and the mathematical modelling of such phenomena. In this article we study the longitudinal vibration of double nanorod system (DNRS). According to the best of the author's knowledge, longitudinal vibration of double nanorod systems using local/ nonlocal continuum models has not been studied. In the present paper small-scale effects on the DNRS are investigated by employing nonlocal continuum mechanics. Governing equations for DNRS are developed using Eringen's nonlocal elasticity theory. An analytical method is developed for solving the nonlocal frequencies of axially vibrating DNRS. Clamped-clamped and clamped-free boundary conditions are employed in and their explicit relationships have been derived. It has been shown that the nonlocal effect is quite significant in the longitudinal vibration studies and needs to be included in the continuum model of DNRS. The present study could bring in helpful insights while investigating multiple-nanorod-system-models for nanosensor applications and in NOMS.

\section{Review of nonlocal rod theory}

The basic equations for a linear homogenous nonlocal elastic body neglecting the body forces can be written as [8-24]

$$
\begin{aligned}
& \sigma_{i j, j}=0, \\
& \sigma_{i j}(x)=\int \phi\left(\left|x-x^{\prime}\right|, \alpha\right) H_{i j k l} \varepsilon_{k l}\left(x^{\prime}\right) d V\left(x^{\prime}\right), \quad \forall x \in V \\
& \varepsilon_{i j}=\frac{1}{2}\left(u_{i, j}+u_{j, i}\right)
\end{aligned}
$$

The terms $\sigma_{i j}, \varepsilon_{k l}$ and $H_{i j k l}$ are the stress, strain and fourth-order elasticity tensors, respectively. The above equation (Eq. (1)) couples the stress due to nonlocal elasticity and the stress due to classical elasticity. The kernel function $\phi\left(\left|x-x^{\prime}\right|, \alpha\right)$ is the nonlocal modulus. The nonlocal modulus acts as an attenuation function incorporating into constitutive equations the nonlocal 
effects at the reference point $x$ produced by local strain at the source $x^{\prime}$. The term $\left|x-x^{\prime}\right|$ represents the distance in the Euclidean form and $\alpha$ is a material constant that depends on the internal (e.g. lattice parameter, granular size, distance between the $\mathrm{C}-\mathrm{C}$ bonds) and external characteristics lengths (e.g. crack length, wave length). Material constant $\alpha$ is defined as $\alpha=e_{0} a / \ell$. Here $e_{0}$ is a constant for calibrating the model with experimental results and other validated models [8]. The parameter $e_{0}$ is estimated such that the relations of the nonlocal elasticity model could provide satisfactory approximation to the atomic dispersion curves of the plane waves with those obtained from the atomistic lattice dynamics. $a$ and $\ell$ are the internal (e.g. lattice parameter, granular size, distance between $\mathrm{C}-\mathrm{C}$ bonds) and external characteristics lengths (e.g. crack length, wave length) of the nanostructure.

Eq. (1) is in a partial-integral form and generally difficult to solve analytically. Thus a differential form of the nonlocal elasticity equation is normally used. To understand the transformation of Eq. (1) from integral type into differential type one may see Ref. [8]. Since in this paper we deal with nanorod, we will use the onedimensional differential form of nonlocal elastic equation. The nanorod is assumed to be slender. In the one-dimensional form, the constitutive relation (1) can be reduced to as [14]

$\sigma(x)-\left(e_{0} a\right)^{2} \sigma^{\prime \prime}(x)=E \varepsilon(x)$

where the symbol ( $)^{\prime \prime}$ in Eq. (2) denotes the derivation operator $\partial^{2} / \partial x^{2}$. The term $E$ is the conventional Young's modulus of the nanostructure component (nanorod, nanobeam).

We represent the following stress resultant $N$ as

$N=\int_{A} \sigma(x) d A$

where $A$ is the cross-sectional area of the nanorod. Coupling Eqs. (2) and (3) yield the following nonlocal stress resultant expression

$N-\left(e_{0} a\right)^{2} N^{\prime \prime}(x)=E A u^{\prime}(x, t)$

Consider a slender nanorod of length $L$. The Euler-Lagrange equation (the equation of motion) for an axially vibrating nanorod in the domain $0<x<L$ can be expressed as

$N^{\prime}+f(x, t)=m \ddot{u}(x, t)$

where $\ddot{u}$ implies the time derivative $\partial^{2} / \partial t^{2}$. Here $m$ and $u(x, t)$ are the mass and axial displacements of the nanorod and $f(x, t)$ is the axially distributed force. Using Eqs. (4) and (5) we obtain the expression for the axially vibrating nanorod as $[12,14,15]$

$-E A u^{\prime \prime}(x, t)-f(x, t)+\left(e_{0} a\right)^{2} f^{\prime \prime}(x, t)+m_{1} \ddot{u}_{1}(x, t)-\left(e_{0} a\right)^{2} m_{1} \ddot{u}_{1}^{\prime \prime}(x, t)=0$

Next we utilize Eq. (6) for the development of coupled doublenanorod system.

\section{Equations of motion of double-nanorod-system (DNRS)}

Consider a double nanorod system (DNRS) as shown in Fig. 2. The nanorods (nanorod- 1 and nanorod-2) are considered to be of length $L$. Axially distributed springs attach the two nanorods. The springs are used to substitute the effect of elastic medium, forces due to nanooptomechanical effect or van der Waals forces (vdW) between the two nanorods. The springs are assumed to have a stiffness, $k$. In general, the two nanorods are different where the length, mass per unit length and axial rigidity of the $i$ th rod are $L_{i}$, $m_{i}$ and $E_{i} A_{i}(i=1,2)$, respectively. These parameters are assumed a

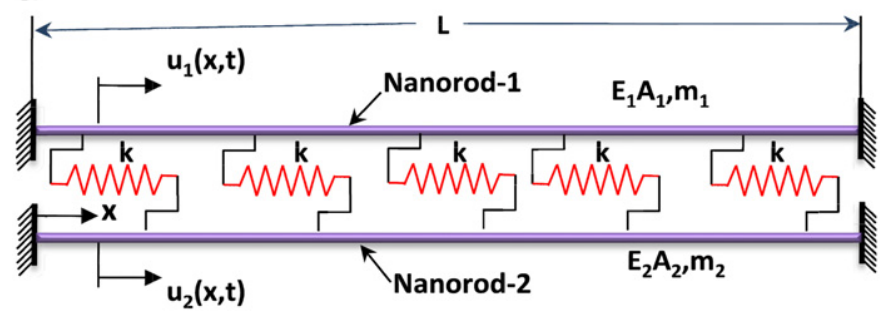

b

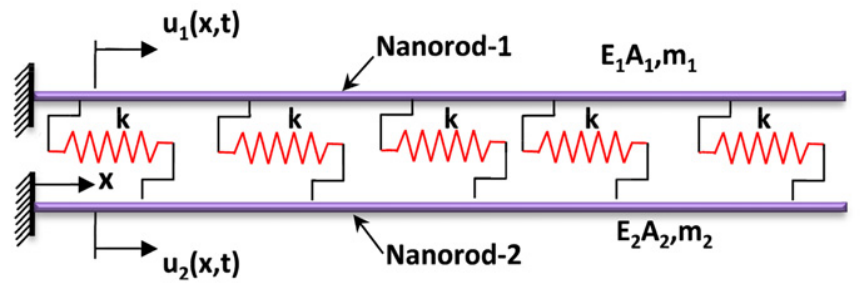

Fig. 2. Double nanorod configuration. (a) Clamped-clamped boundary condition and (b) clamped-free (cantilever) boundary condition.

to be constant along each nanorod. The longitudinal displacements over the two nanorods are denoted by $u_{1}(x, t)$ and $u_{2}(x, t)$ (Fig. 2).

Using the nonlocal continuum rod theory (Eq. (6)), the governing equations for the longitudinal vibration of DNRS can be expressed as

$$
\begin{aligned}
\text { nanorod-1: } & -E_{1} A_{1} u^{\prime \prime}{ }_{1}(x, t)+k\left[u_{1}(x, t)-u_{2}(x, t)\right]-\left(e_{0} a\right)^{2} k\left[u^{\prime \prime}{ }_{1}(x, t)\right. \\
& \left.-u^{\prime \prime}{ }_{2}(x, t)\right]+m_{1} \ddot{u}_{1}(x, t)-\left(e_{0} a\right)^{2} m_{1} \ddot{u}_{1}^{\prime \prime}(x, t) \\
& =f(x, t)-\left(e_{0} a\right)^{2} f^{\prime \prime}(x, t)
\end{aligned}
$$

nanorod-2: $\quad-E_{2} A_{2} u^{\prime \prime}{ }_{2}(x, t)-k\left[u_{1}(x, t)-u_{2}(x, t)\right]+\left(e_{0} a\right)^{2} k\left[u^{\prime \prime}{ }_{1}(x, t)\right.$ $\left.-u_{2}^{\prime \prime}(x, t)\right]+m_{2} \ddot{u}_{2}(x, t)-\left(e_{0} a\right)^{2} m_{2} \ddot{u}_{2}^{\prime \prime}(x, t)=0$

where $x$ is the axial position along the nanorods. Dots $(\cdot)$ and primes (") denote partial derivatives with respect to time $t$ and position coordinate $x$, respectively. With a simple manipulation of variables, the equations can be uncoupled and general analysis can be used to determine the solutions.

$E_{1} A_{1}=E_{2} A_{2}=\bar{e} \equiv$ constant

$m_{1}=m_{2}=m \equiv$ constant

$f(x, t)=0$ (free-vibration)

where $\bar{e}$ and $m$ denote the axial rigidity and mass per unit length, respectively. It should be noted that $\bar{e}$ and $e_{0}$ are different and should not be confused.

Considering Eqs. (7) and (8) and the assumptions from Eqs. (9)-(11), we get

nanorod-1: $\quad-\bar{e} u_{1}^{\prime \prime}(x, t)+k\left[u_{1}(x, t)-u_{2}(x, t)\right]-\left(e_{0} a\right)^{2} k\left[u^{\prime \prime}{ }_{1}(x, t)\right.$

$$
\left.-u^{\prime \prime}{ }_{2}(x, t)\right]+m \ddot{u}_{1}(x, t)-\left(e_{0} a\right)^{2} m \ddot{u}_{1}^{\prime \prime}(x, t)=0
$$

nanorod-2: $\quad-\bar{e} u^{\prime \prime}{ }_{2}(x, t)-k\left[u_{1}(x, t)-u_{2}(x, t)\right]+\left(e_{0} a\right)^{2} k\left[u^{\prime \prime}{ }_{1}(x, t)\right.$

$$
\left.-u^{\prime \prime}{ }_{2}(x, t)\right]+m \ddot{u}_{2}(x, t)-\left(e_{0} a\right)^{2} m_{1} \ddot{u}_{2}{ }_{2}(x, t)=0
$$

For the DNRS, we denote

$u(x, t)=u_{1}(x, t)-u_{2}(x, t)$, 
such that

$u_{1}(x, t)=u(x, t)+u_{2}(x, t)$

Here $u(x, t)$ is the relative displacement of the primary nanorod with respect to the secondary nanorod. Using the definitions in Eqs. (14) and (15) and substituting in Eqs. (12) and (13) we obtain $-\bar{e} u^{\prime \prime}(x, t)+2 k u(x, t)-2\left(e_{0} a\right)^{2} k u^{\prime \prime}(x, t)+m \ddot{u}(x, t)-\left(e_{0} a\right)^{2} m \ddot{u}{ }^{\prime \prime}(x, t)=0$

$-\bar{e} u^{\prime \prime}{ }_{2}(x, t)+m \ddot{u}_{2}(x, t)-\left(e_{0} a\right)^{2} m \ddot{u}^{\prime \prime}{ }_{2}(x, t)=k u(x, t)-\left(e_{0} a\right)^{2} k u^{\prime \prime}(x, t)$

when the nonlocal effects are ignored $\left(e_{0} a=0\right)$ and a single rod is considered, the above equations revert to the equations of classical rod theory [36]. For the present analysis of coupled DNRS, we will be primarily dealing with Eq. (16).

\section{Longitudinal vibration of nonlocal double-nanorod system}

\subsection{Solution methodology}

Assuming that the relative motion $u(x, t)$ is one of its natural modes of vibration, the general solution of Eq. (16) can be written as $u(x, t)=U(x) e^{i \omega t}$

where $\omega$ is the natural frequency and $U(x)$ the corresponding mode shape of the DNRS.

Substituting Eq. (18) into Eq. (16) we obtain

$U^{\prime \prime}(x)+\beta^{2} U(x)=0$

where

$\beta^{2}=\frac{m \omega^{2}-2 k}{\bar{e}+2\left(e_{0} a\right)^{2} k-\left(e_{0} a\right)^{2} m \omega^{2}}$

\subsection{Clamped-clamped boundary condition}

Now we assume that all the ends of the DNRS are clamped (Fig. 2a). Solution of Eq. (19) can be expressed in the form

$U(x)=A_{1} \cos \beta x+A_{2} \sin \beta x$

The boundary conditions of clamped-clamped case (for both nanorods) are given as

nanorod-1: $\quad u_{1}(0, t)=0 ; \quad u_{1}(L, t)=0$

nanorod-2: $\quad u_{2}(0, t)=0 ; \quad u_{2}(L, t)=0$

It should be noted that in the present case (all ends clamped) the local and nonlocal boundary conditions are equivalent.

Using Eq. (14) we obtain

$u(0, t)=u_{1}(0, t)-u_{2}(0, t)$,

$u(L, t)=u_{1}(L, t)-u_{2}(L, t)$,

And using Eq. (18) we obtain

$U(0)=0, \quad U(L)=0$

Substituting Eq. (26) into Eq. (21) yields the following expression:

$A_{1}=0, \quad A_{2} \sin \beta L=0$,

Therefore the frequency equation is $\sin \beta L=0$, and their roots are

$\beta L=r \pi, \quad r=1,2 \ldots$

The combination of Eqs. (20) and (29) leads to

$\frac{m \omega^{2}-2 k}{\bar{e}+2\left(e_{0} a\right)^{2} k-\left(e_{0} a\right)^{2} m \omega^{2}}=\left(\frac{r \pi}{L}\right)^{2}$

From Eq. (30) the natural frequency can be obtained explicitly as

$(\omega)_{r}=\sqrt{\frac{(\pi r / L)^{2}\left(\bar{e}+2 k\left(e_{0} a\right)^{2}\right)+2 k}{m\left(1+\left(e_{0} a\right)^{2}(\pi r / L)^{2}\right)}}, \quad r=1,2 \ldots$

The ratio of the local and nonlocal longitudinal natural frequencies of the DNRS can be given as

Ratio $=\left(\frac{\omega_{N L}}{\omega_{L}}\right)_{r}=\sqrt{\frac{1}{1+\left(e_{0} a / L\right)^{2}(\pi r)^{2}}\left(1+\frac{2\left(e_{0} a / L\right)^{2}(\pi r)^{2}}{\bar{e} / k L^{2}(\pi r)^{2}+2}\right)}, \quad r=1,2 \ldots$

\subsection{Clamped-free (cantilever) boundary condition}

The boundary conditions of the clamped-free case (for both nanorods) are given as

nanorod-1: $\quad u_{1}(0, t)=0 ; \quad N_{1}(L, t)=\bar{e} u_{1}^{\prime}(L, t)+m\left(e_{0} a\right)^{2} \ddot{u}^{\prime}{ }_{1}$

$$
+\left(e_{0} a\right)^{2} k\left[u_{1}^{\prime}(L, t)-u_{2}^{\prime}(L, t)\right]=0
$$

nanorod-2 $: \quad u_{2}(0, t)=0 ; \quad N_{2}(L, t)=\bar{e} u_{2}^{\prime}(L, t)+m\left(e_{0} a\right)^{2} \ddot{u}_{2}^{\prime}$

$$
-\left(e_{0} a\right)^{2} k\left[u_{1}^{\prime}(L, t)-u_{2}^{\prime}(L, t)\right]=0
$$

Using Eq. (14) we write

$u(0, t)=u_{1}(0, t)-u_{2}(0, t)=0$,

$N_{1}(L, t)-N_{2}(L, t)=\bar{e} u_{1}^{\prime}(L, t)+m\left(e_{0} a\right)^{2} \ddot{u}^{\prime}{ }_{1}+\left(e_{0} a\right)^{2} k\left[u^{\prime}{ }_{1}(L, t)-u^{\prime}{ }_{2}(L, t)\right]$

$$
-\bar{e} u_{2}^{\prime}(L, t)-m\left(e_{0} a\right)^{2} \ddot{u}_{2}^{\prime}+\left(e_{0} a\right)^{2} k\left[u_{1}^{\prime}(L, t)-u_{2}^{\prime}(L, t)\right]=0
$$

Using Eqs. (14) and (18), Eqs. (35) and (36) reduce to $U(0)=0, \quad U^{\prime}(L)=0$

It should be noted that for the present case (C-F) the local and nonlocal boundary conditions reduce to similar expressions.

Substituting Eq. (37) into Eq. (21) one obtains

$A_{1}=0, \quad A_{2} \cos \beta L=0$,

Therefore the frequency equation is

$\cos \beta L=0$,

The roots of this transcendental equation are

$\beta L=(2 r-1) \frac{\pi}{2}, \quad r=1,2 \ldots$

From Eq. (40) we get

$\frac{m \omega^{2}-2 k}{\bar{e}+2\left(e_{0} a\right)^{2} k-\left(e_{0} a\right)^{2} m \omega^{2}}=\left[(2 r-1) \frac{\pi}{2 L}\right]^{2}$ 
Thus the natural frequency of the clamped-free DNRS is given by

$(\omega)_{r}=\sqrt{\frac{[(2 r-1) \pi / 2 L]^{2}\left(\bar{e}+2 k\left(e_{0} a\right)^{2}\right)+2 k}{m\left(1+\left(e_{0} a\right)^{2}[(2 r-1) \pi / 2 L]^{2}\right)}}, \quad r=1,2$

The ratio of the local and nonlocal longitudinal natural frequencies of the system is given by

Ratio $=\left(\frac{\omega_{N L}}{\omega_{L}}\right)_{r}=\sqrt{\frac{1}{1+\left(e_{0} a / L\right)^{2}[(2 r-1) \pi / 2]^{2}}\left(1+\frac{2\left(e_{0} a / L\right)^{2}[(2 r-1) \pi / 2]^{2}}{\bar{e} / k L^{2}[(2 r-1) \pi / 2]^{2}+2}\right)}$,

$r=1,23$,

\section{Longitudinal vibration of auxiliary (secondary) nanorod}

Using the vibration analysis as described previously, the longitudinal vibration of the secondary nanorod (nanorod-2) can be obtained. For the longitudinal vibration of the secondary nanorod, the relative axial displacements between the two nanorods are absent, that is $\left(u_{1}-u_{2}=0\right)$. We apply the same procedure for solving Eq. (17).

\subsection{Clamped-clamped boundary conditions}

The eigen values for the all-ends-clamped boundary conditions are obtained as

$\beta_{2 r}=\frac{m \omega^{2}}{\bar{e}-\left(e_{0} a\right)^{2} m \omega^{2}}=\left(\frac{r \pi}{L}\right)^{2}$

From this, the natural frequencies can be obtained as

$\omega_{2 r}=\sqrt{\frac{[r \pi / L]^{2} \bar{e}}{m\left(1+\left(e_{0} a\right)^{2}[r \pi / L]^{2}\right)}}$

The ratio of the nonlocal and local frequencies is given by

Ratio $=\left(\frac{\omega_{N L}}{\omega_{L}}\right)_{2 r}=\sqrt{\frac{1}{1+\left(e_{0} a / L\right)^{2}(r \pi)^{2}}}$

\subsection{Clamped-free boundary conditions}

The Eigen-values for the clamped-free boundary conditions for both nanorods are given as

$\beta_{2 r}=\frac{m \omega^{2}}{\bar{e}-\left(e_{0} a\right)^{2} m \omega^{2}}=\left[\frac{(2 r-1) \pi}{2 L}\right]^{2}$

The natural frequencies for the clamped-free boundary condition are given as

$\omega_{2 r}=\sqrt{\frac{[(2 r-1) \pi / 2 L]^{2} \bar{e}}{m\left(1+\left(e_{0} a\right)^{2}[(2 r-1) \pi / 2 L]^{2}\right)}}$

The ratio of the nonlocal and local frequencies is obtained as

Ratio $=\left(\frac{\omega_{N L}}{\omega_{L}}\right)_{2 r}=\sqrt{\frac{1}{1+\left(e_{0} a / L\right)^{2}[(2 r-1) \pi / 2]^{2}}}$

The frequency ratios in Eqs. (46) and (49) turn out to be the same as that of the axial vibration of a single nanorod [14] for the corresponding boundary conditions. Here it should be interpreted that the DNRS vibrates in-phase motion; and that the whole DNRS can be considered as a single nanorod. For the in-phase type vibration, the DNRS would become independent of the effect of coupling medium (springs, attractive van der Waal forces and Langevin forces).

\section{Numerical results for double-carbon-nanotube-rod system}

The nonlocal theory for DNRS developed here is a generalised theory and can be applied for the longitudinal vibration analysis of coupled carbon nanotubes [34,35], double ZnO nanorods systems [4] and double-nanobeam-systems for NOMS application [37,38].

The results for longitudinal vibration of double-nanorod systems obtained using the nonlocal elasticity theory is compared with the previous classical solutions [39]. In Ref. [39] vibration of scale-free double-rod-system is studied. For the comparative study we consider the following arbitrary values, $L=1 \mathrm{~nm}, m=10^{-9} \mathrm{~kg} / \mathrm{m}$, $\bar{e}=1 \mathrm{nN}$ and $e_{0} a=0-2 \mathrm{~nm}$. Both low and high stiffness values of the couplings springs are considered, viz. $K=8 \mathrm{~N} / \mathrm{nm}$ and $80 \mathrm{~N} / \mathrm{nm}$, respectively. Here it should be noted that similar numerical magnitudes were taken in Ref. [39], but length was in larger scale (in metres). From Tables 1 and 2 we can see that the small-scale effects are truly prominent for double-nanorod systems, which is not reflected for classical double-rod-system.

For further realistic study, we consider the properties of the nanorod as that of a single-walled carbon nanotube (SWCNT) [40]. An armchair SWCNT with chirality $(5,5)$ is considered. The radius of each individual nanorod (SWCNT) is assumed as $0.34 \mathrm{~nm}$. Young's modulus, E, is considered as 0.971 TPa [40]. Here the term DNRS would denote the case of double-carbon-nanotube-rod system.

To study the influence of the small length scale (nonlocal effects) on the longitudinal vibration of DNRS, frequency ratio is analysed for different (i) lengths, (ii) scale coefficients or nonlocal parameter, (iii) boundary conditions and (iv) modes of vibration. Both clamped-clamped and clamped-free boundary conditions (Fig. 2) are employed in the study. Different values of length of the nanorod are considered for the computation. Lengths are taken in the range $L=2-30 \mathrm{~nm}$. Both the nanotubes (nanorod-1 and nanorod-2) are assumed to have the same geometrical and material properties.

\subsection{Effect of the nonlocal parameter in the clamped-type DNRS}

Fig. 3 depicts the frequency ratio percent (FRP) versus the length of the DNRS for different nonlocal parameter $\left(e_{0} a\right)$.

Table 1

Comparison of first four natural frequencies $(\mathrm{GHz})$ of double nanorod system with classical solutions with stiffness, $K=8 \mathrm{~N} / \mathrm{nm}$.

\begin{tabular}{llllll}
\hline & $e_{0} a=0.5 \mathrm{~nm}$ & $e_{0} a=1.0 \mathrm{~nm}$ & $e_{0} a=1.5 \mathrm{~nm}$ & $e_{0} a=2.0 \mathrm{~nm}$ & Classical [39] \\
\hline 1 & 1.2353 & 0.8436 & 0.6137 & 0.4764 & 1.5708 \\
2 & 4.1864 & 4.088 & 4.0468 & 4.0283 & 4.2974 \\
3 & 1.8411 & 0.9782 & 0.6601 & 0.4972 & 4.7124 \\
4 & 4.4033 & 4.1179 & 4.0541 & 4.0308 & 6.1812 \\
\hline
\end{tabular}

Table 2

Comparison of first four natural frequencies $(\mathrm{GHz})$ of double nanorod system with classical solutions; stiffness, $K=80 \mathrm{~N} / \mathrm{nm}$.

\begin{tabular}{cccccc}
\hline & $e_{0} a=0.5 \mathrm{~nm}$ & $e_{0} a=1.0 \mathrm{~nm}$ & $e_{0} a=1.5 \mathrm{~nm}$ & $e_{0} a=2.0 \mathrm{~nm}$ & Classical [39] \\
\hline 1 & 1.2353 & 0.8436 & 0.6137 & 0.4764 & 1.5708 \\
2 & 12.7093 & 12.6772 & 12.664 & 12.6581 & 12.7463 \\
3 & 1.8411 & 0.9782 & 0.6601 & 0.4972 & 4.7124 \\
4 & 12.7824 & 12.6869 & 12.6663 & 12.6589 & 13.4984 \\
\hline
\end{tabular}




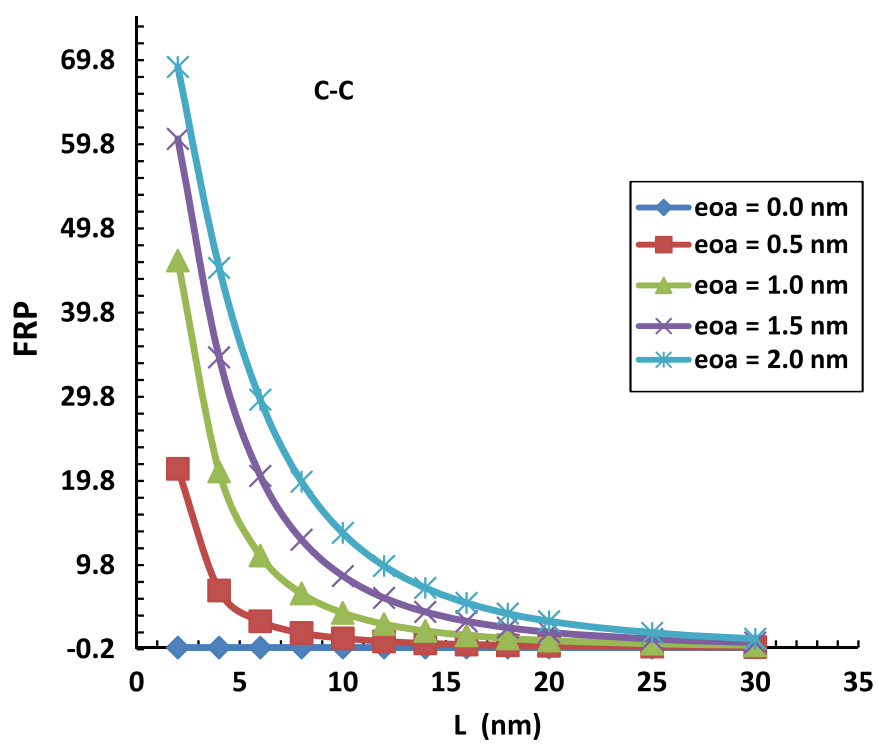

Fig. 3. Variation in frequency ratio percent with length of nanorods in DNRS for different nonlocal parameters and clamped-clamped boundary condition.

Frequency ratio percent (FRP) is defined as

$\mathrm{FRP}=(1-$ Ratio $) \times 100$

where Ratio is defined as the ratio of nonlocal frequency to local frequency $\left(\omega_{N L} / \omega_{L}\right)$. All the four-boundary condition of the DNRS in this figure is considered to be clamped (C). For the choice of the nonlocal parameter we take, $e_{0} a=0.0,0.5,1.0,1.5$ and $2.0 \mathrm{~nm}$. Similar range of values was assumed by Wang et al. [11], Aydogdu [14], Heireche et al. [16], Pradhan and Murmu [23,24], Murmu and Pradhan [21,42,43] and Duan and Wang [41]. One can see that when $\left(e_{0} a=0\right)$ the nonlocal rod model reverts to the classical rod theory. The axially distributed springs have been assumed a stiffness value of $k=2 \mathrm{~N} / \mathrm{nm}$. The stiffness of the axially distributed springs will depend on the stiffness of the chosen elastic medium enclosed between the nanorods or atomistic forces. If there is no intermediate elastic medium, the twin nanorods will be subjected to van der Waals forces or Langevin forces $[37,38]$. The stiffness of the springs will then be depending on the perpendicular distance between the two nanorods. The present following discussion will be on vibration of DNRS based on Section 4.

From Fig. 3, it is observed that the nonlocal frequency solution of DNRS is smaller than the classical (local) frequency solution. This is attributed to the effect of small length scale. In addition, increase in the nonlocal parameter decreases the natural frequency (increases the FRP). This can be accounted by the fact that the nonlocal parameter leads to a decrease in the overall stiffness of DNRS. The small-scale effects make the DNRS 'softer'. As the length of the nanorods in DNRS increases, the FRP decreases. This is because with increase in length, the influence of nonlocal effect reduces. With further increase in length the curves drop and become flat in nature. At larger lengths there is no difference between the frequencies by the local and nonlocal rod models. Approximately at $L \geq 30 \mathrm{~nm}$ all results converge to the local frequency $\left(e_{0} a=0\right)$. This implies that the nonlocal effect diminishes with increase in the DNRS length and practically disappears after a certain length. Similar findings are reported by other authors [14]. This may be interpreted by the fact that the wavelength increases with an increase in the length, which in turn decreases the effect of the small length scale.

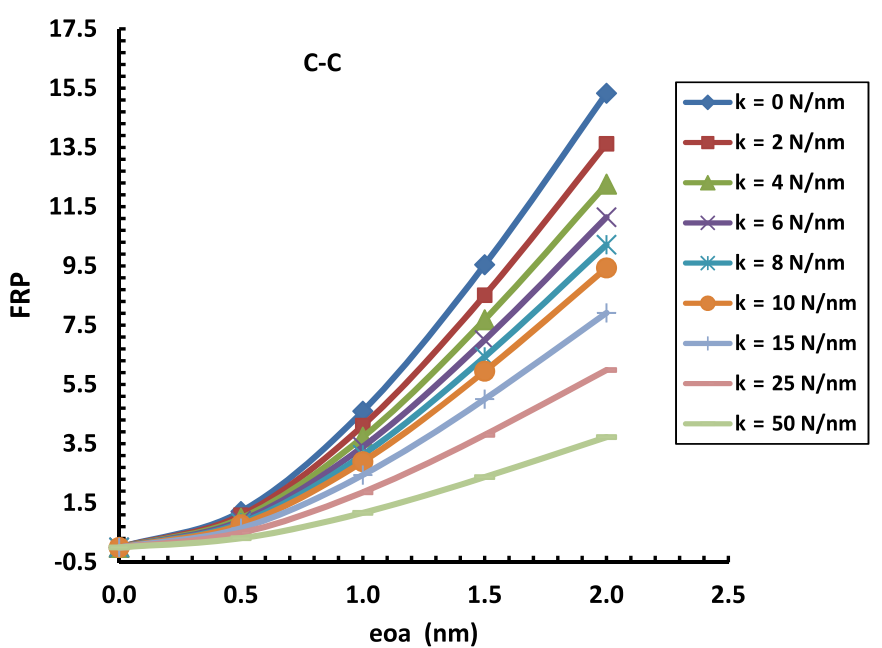

Fig. 4. Variation in frequency ratio percent with nonlocal parameter for different stiffnesses of springs and clamped-clamped boundary condition in DNRS.

\subsection{Effect of the coupling spring stiffness in DNRS}

To show the effect of the stiffness of the springs on the longitudinal vibration of DNRS, FRP versus nonlocal parameter $e_{0} a$ for various stiffness values has been plotted and shown in Fig. 4 . The stiffness of the springs are assumed in the range $k=0-50 \mathrm{~N} / \mathrm{nm}$. The figure shows that as the nonlocal parameter increases, the FRP increases for all stiffness of the springs considered. This implies that the natural frequencies of the DNRS decrease with increase in nonlocal effects (same as Fig. 3). It is noticed that with increase in value of the spring stiffness, the FRP decreases (i.e., frequency increases). It is interesting to note that nonlocal effects are more pronounced in nanorod without the presence of the springs or DNRS in in-phase type vibration compared to strongly coupled nanorods (in out-of-phase vibration). The stiffness of the coupling springs has a subduing effect on the small-scale effects for longitudinal vibrating DNRS. This behaviour may be very important in studying the optical effects in double-nanobeams system in NOMS $[37,38]$.

\subsection{Effect of higher modes of vibration in DNRS}

To show the effect of higher modes in the longitudinal vibration of DNRS, FRP versus the nonlocal parameter for the first eight natural frequencies are plotted and shown in Fig. 5. The term $m(1)$ represents the first modes with two natural frequencies. The first lowest natural frequency $m(1)=1$ is the frequency of the secondary nanorod (or double nanorod acting as single nanorod $\left.\left(u_{1}-u_{2}=0\right)\right)$. This happens when the DNRS is vibrating in-phase motion. The second natural frequency $m(1)=2$ is from the DNRS when $\left(u_{1}-u_{2} \neq 0\right)$. This happens for the case when DNRS vibrates in out-of-phase motion.

It is observed from Fig. 5 that, with increase in the nonlocal parameter, the value of FRP increases. This effect is more seen for the higher modes of longitudinal vibration. Thus, the nonlocal effects for the longitudinal vibration of DNRS are more pronounced in higher modes. It should be noted that the first frequency of each modes are independent of the stiffness of the coupling springs (due to axial in-phase vibration).

6.4. Effect of nonlocal parameter, spring stiffness and higher modes in cantilever-type-DNRS

Figs. 6-8 show the variation in FRP for the cantilevertype-DNRS, analogous to that described in Figs. 3-5 for the 


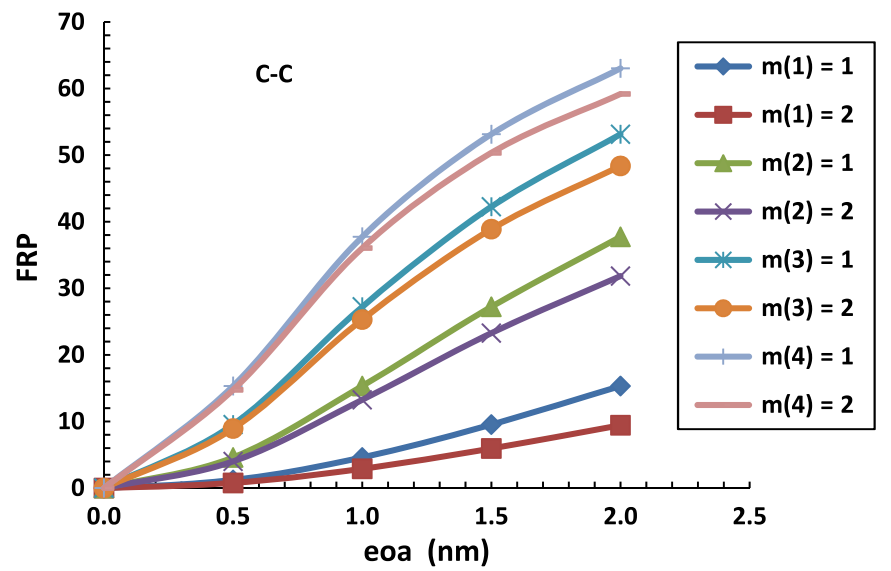

Fig. 5. Variation in frequency ratio percent with nonlocal parameter for first eight modes of vibration and clamped-clamped boundary condition.

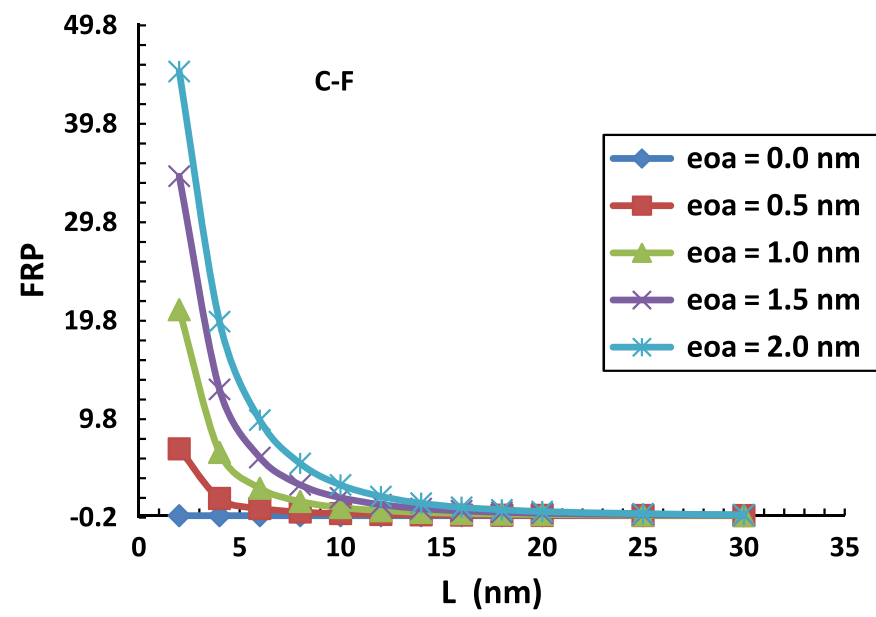

Fig. 6. Variation in frequency ratio percent with length of nanorods in DNRS for different nonlocal parameters and clamped-free boundary condition.

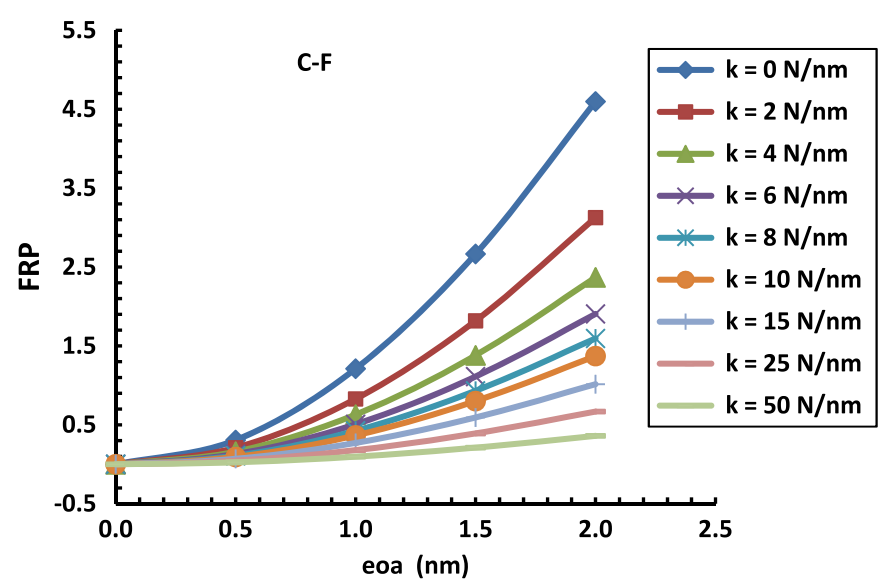

Fig. 7. Variation in frequency ratio percent with nonlocal parameter for different stiffnesses of springs and clamped-free boundary condition in DNRS.

clamped-type DNRS. Figs. 6-8 show the plot for clamped-free boundary conditions for both nanorod-1 and nanorod-2. It can be observed from Fig. 6 that with increase in the length of DNRS, the FRP decreases. Approximately at $L \geq 20 \mathrm{~nm}$ all results converge to the local frequency $\left(e_{0} a=0\right)$. It is thus concluded that the

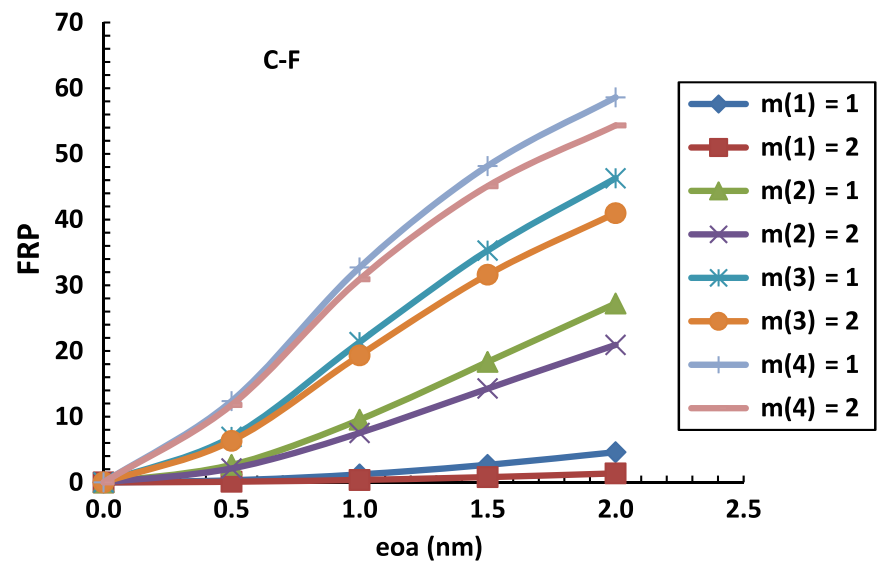

Fig. 8. Variation in frequency ratio percent with nonlocal parameter for first eight modes of vibration and clamped-free boundary condition.

convergence of local and nonlocal models is faster (with respect to length) in the cantilever boundary condition than in the clamped-clamped boundary condition. In addition, it is also noted that with increase in nonlocal parameter, the natural longitudinal frequency of the DNRS decreases. This nonlocal frequency results are opposite to that of the transverse vibration of nanobeam with cantilever boundary conditions [44]. In nanobeam, fundamental frequencies increase with increase in nonlocal parameter while in nanorod fundamental frequencies decrease with increase in nonlocal parameter.

In Fig. 7 the FRP is plotted against the nonlocal parameter for the clamped free boundary conditions. Similar variation in results as that in Fig. 4 can be observed. The stiffness of the springs has a subduing effect on the small-scale effects. From Fig. 8, it is seen that the nonlocal effect in the clamped-free boundary condition is more prominent for the higher modes of longitudinal vibration than in the all-clamped DNRS.

\section{Conclusions}

A nonlocal rod model for the longitudinal vibration of doublenanorod-system (DNRS) is developed based on Eringen's nonlocal continuum mechanics. In this model the small-scale effects are explicitly taken into account. An exact analytical method is proposed for solving the nonlocal frequencies of axially vibrating DNRS. Clamped-clamped and clamped-free boundary conditions are employed and their explicit relationships have been derived. It is found that the longitudinal vibration frequencies of DNRS are over estimated by the classical (local) nanorod model as it disregards the effect of the small-length scale. It is found that the stiffness of the coupling springs in DNRS has a subduing effect on the small-scale effects. Unlike the transverse vibration of nanobeams, the fundamental natural frequency of axially vibrating nanorods has a decreasing nature with the increasing nonlocal parameter. Similar to the transverse vibration of nanobeams, the nonlocal effects in longitudinal vibration of DNRS are more pronounced in the higher modes of vibration. The present study may bring in helpful insights while investigating multiplenanorod-system-models for future NOMS applications.

\section{References}

[1] H. Dai, J.H. Hafner, A.G. Rinzler, D.T. Colbert, R.E. Smalley, Nature 384 (1996)

[2] A. Bachtold, P. Hadley, T. Nakanishi, C. Dekker, Science 294 (2001) 1317. 
[3] P. Kim, C.M. Lieber, Science 286 (1999) 2148.

[4] J.G. Wen, J.Y. Lao, D.Z. Wang, T.M. Kyaw, Y.L. Foo, Z.F. Ren, Chem. Phys. Lett. 372 (2003) 717.

[5] R.S. Wagner, in: A.P. Levitt (Ed.), Whisker Technology, Wiley-Interscience, New York, 1970.

[6] W.I. Park, D.H. Kim, S.W. Jung, G.C. Yi, Appl. Phys. Lett. 80 (2002) 4232.

[7] J.H. Choy, E.S. Jang, J.H. Won, J.H. Chung, D.J. Jang, Y.W. Kim, Appl. Phys. Lett. 84 (2004) 287.

[8] A.C. Eringen, J. Appl. Phys. 54 (1983) 4703.

[9] J. Peddieson, G.G. Buchanan, R.P. McNitt, Int. J. Eng. Sci. 41 (1972) 305.

[10] P. Lu, J. Appl. Phys. 101 (2007) 073504

[11] C.M. Wang, Y.Y. Zhang, S.S. Ramesh, S. Kitipornchai, J. Phys. D 39 (2006) 3904

[12] J.N. Reddy, S.D. Pang, J. Appl. Phys. 103 (2008) 023511.

[13] C.M. Wang, W.H. Duan, J. Appl. Phys. 104 (2008) 014303

[14] M. Aydogdu, Physica E 41 (2009) 861.

[15] M. Aydogdu, Physica E 41 (2009) 1651

[16] H. Heireche, A. Tounsi, A. Benzair, M. Maachou, E.A. Adda Bedia, Physica E 40 (2008) 2791.

[17] L. Wang, Physica E 41 (2009) 1835.

[18] Y. Yan, W.Q. Wang, L.X. Zhang, Appl. Math. Modelling 34 (11) (2010) 3422.

[19] F. Khademolhosseini, R.K.N.D. Rajapakse, A. Nojeh, Comput. Mater. Sci. 48 (4) (2010) 736.

[20] J. Yang, L.L. Ke, S. Kitipornchai, Physica E 42 (5) (2010) 1727.

[21] T. Murmu, S.C. Pradhan, J. Appl. Phys. 106 (2009) 104301.

[22] T. Murmu, S.C. Pradhan, Mech. Res. Commun. 36 (8) (2009) 933.

[23] S.C. Pradhan, T. Murmu, Comput. Mater. Sci. 47 (1) (2010) 268

[24] S.C. Pradhan, T. Murmu, Physica E 42 (5) (2010) 1293.
[25] J.A. Ruud, T.R. Jervis, F. Spaepan, J. Appl. Phys. 75 (1994) 4969.

[26] E.W. Wong, P.E. Sheehan, C.M. Lieber, Science 277 (1997) 1971

[27] J. Gaspar, V. Chu, J.P. Conde, Appl. Phys. Lett. 84 (2004) 622.

[28] C.T. Sun, H. Zhang, J. Appl. Phys. 93 (2003) 1212.

[29] R. Chowdhury, S. Adhikari, C.W. Wang., F. Scarpa, Comput. Mater. Sci. 48 (4) (2010) 730.

30] Y. Chen, J.D. Lee, A. Eskandarian, Int. J Solids Struct. 41 (2004) 2085.

[31] S. Filiz, M. Aydogdu, Comput. Mater. Sci. 3 (2010) (2010) 619493 (2010) (2010) 619.

[32] T. Murmu, S.C. Pradhan, Physica E 41 (8) (2009) 1628.

[33] S. Sapmaz, P. Jarillo-Herrero, Ya.M. Blanter, C. Dekker, H.S.J. van der Zant, Phys. Rev. Lett. 96 (2006) 026801.

[34] R. Chowdhury, C.Y. Wang, S. Adhikari, F.M. Tong, Physica E . doi:10.1016/ j.physe.2010.05.003 corrected proof

[35] R. Chowdhury, C.Y. Wang, S. Adhikari, J. Phys. D: Appl. Phys. 43 (085405) (2010) 11

[36] S. Timoshenko, Vibration Problems in Engineering, second ed., New York (1937).

[37] Q. Lin, J. Rosenberg, D. Chang, R. Camacho, M. Eichenfield, K.J. Vahala, O. Painter, Nat. Photonics 4 (2010) 236.

[38] M. Eichenfield, R. Camacho, J. Chan, K.J. Vahala, O. Painter, Nature 459 (2009) 550.

[39] H. Erol, M. Gurgoze, J. Sound Vib. 276 (2004) 419.

[40] J.P. Lu, Phys. Rev. Lett. 79 (7) (1997).

[41] W.H. Duan, C.M. Wang, Nanotechnology 18 (2007) 385704

[42] T. Murmu, S.C. Pradhan, Physica E 41 (7) (2009) 1232.

[43] T. Murmu, S.C. Pradhan, Comput. Mater. Sci. 46 (4) (2009) 854-859.

[44] P. Lu, H.P. Lee, C. Lu, P.Q. Zhang, J. Appl. Phys. 99 (2006) 073510. 